\title{
Temozolomide in malignant glioma
}

This article was published in the following Dove Press journal:

OncoTargets and Therapy

20 July 2010

Number of times this article has been viewed

\section{Gregor Dresemann}

Center for Neurooncology at Aerztehaus Velen, Velen, Germany

Correspondence: Gregor Dresemann

Center for Neurooncology at

Aerztehaus Velen, Ignatiusstrasse

8, 46342 Velen, Germany

Tel +492863923300

Fax +492863923319

Email onkologie@wirsindeinteam.de
Abstract: Glioblastoma multiforme WHO grade IV (GBM) is the most aggressive malignant glioma and the most frequent primary tumor of the central nervous system. The median survival of newly diagnosed GBM patients was between 9 to 12 months prior to treatment with temozolomide being introduced. Primary resection that is as complete as possible is recommended for malignant glioma. Conventional fractionated irradiation 55 to $60 \mathrm{gy}$ with concomitant temozolomide followed by standard temozolomide 6 cycles $(5 / 28)$ (EORTC/NCIC-regime published by R Stupp in 2005) is the standard of care for newly diagnosed GBM after surgery, independent of the methylation status of the MGM-T gene promoter. Age is no contraindication for treatment with temozolomide, although comorbidity and performance status have to be considered. For temozolomide naive GBM and astrocytoma grade III patients with disease progression, temozolomide is still the treatment of choice outside of clinical studies. A general consensus regarding the schedule of choice has not yet been achieved; so far the 5 out of 28 days regimen $(5 / 28)$ is the standard of care in most countries. Patients with disease progression after standard temozolomide $(5 / 28)$ are candidates for clinical studies. Outside of clinical studies, dose-dense (7/7), prolonged (21/28), or metronomic (28/28) temozolomide, or alternatively a nitrosourea-based regimen can be an option. The excellent toxicity profile of temozolomide allows for various combinations with antitumor agents. None of these combinations, however, have been demonstrated to be statistically significantly superior compared to temozolomide alone. The role of lower dosed, dose-dense, or continuous regimen with or without drug combination and the role of temozolomide for newly diagnosed astrocytoma grade III and low grade glioma still has to be determined.

Keywords: glioblastoma multiforme, astrocytoma WHO grade III, malignant glioma, temozolomide

Primary tumors of the central nervous system are classified according to the World Health Organization (WHO) guidelines, which were updated in 2007. ${ }^{1}$ Glioblastoma multiforme WHO grade IV (GBM) is one of the most aggressive human malignant diseases and the most frequent primary tumor of the central nervous system with an incidence of 4-5 per 100,000 inhabitants per year in industrialized countries like Europe and the US. The median survival of newly diagnosed GBM patients ranged from 9 to 12 months prior to the introduction of treatment with temozolomide. About two thirds of all GBM cases arise as a primary tumor while one third develops as secondary GBM on basis of prior lower grade malignancies like astrocytoma grade III which shows clear malignant criteria in histopathology and astrocytoma grade II without clear malignant criteria. ${ }^{2}$ For confident histopathological diagnosis a representative specimen 
of the tumor has to be analyzed, and reference pathology is recommended if diagnosis is not clear. Specimens obtained by stereotactical puncture might not be representative of the entire tumor and should be considred to be an exception. Oligodendroglioma grade III is a well defined subtype of astrocytoma grade III and shows better sensitivity to chemotherapy, resulting in an improved median survival. These tumors more often show loss of heterogeneity of chromosome $1 p$ and $19 q$ (LOH 1p19q), which is predictive of a better response to alkylating drugs. ${ }^{3,4}$

Abnormal interactions between macroglia, microglia, a special type of immune competent intracerebral migrating and modulating cells and the microvascular structure appear to play a role in the development of primary brain tumors. ${ }^{5}$

It has been demonstrated that many growth factor receptor families, such as epithelial growth factor receptor (EGF-R), platelet derived growth factor receptor (PDGF-R), and vascular endothelium growth factor receptor (VEGF-R), and their mutations, are involved in pathogenesis of GBM. ${ }^{6-12}$ There is evidence that primary and secondary GBM have a different molecular-genetic basis, however, treatment has not been stratified by these findings so far. ${ }^{2,13-15}$

The methylation status of the O6-methylguaninmethyltransferases (MGM-T) gene promoter is important for upcoming trials and potentially for future treatment strategies. MGM-T is a genome repair enzyme able to repair DNA lesions caused by alkylating drugs. In about 50\% of GBM the MGM-T gene promoter is methylated, resulting in silencing of the gene and lack of MGM-T capacity with an increased sensitivity to alkylating drugs compared to GBM with nonmethylated MGM-T promoter and normal MGM-T level. ${ }^{16}$ This explains why patients presenting a GBM with a methylated MGM-T promoter respond better to chemotherapeutic agents and have a prolonged survival. Neurooncologists all over the world have discussed the impact of methylation of the MGM-T promoter on individualization of treatment, however, consensus recommendations are still missing. ${ }^{17}$ There is evidence in the literature that prolonged or continuous treatment with alkylating drugs could subsequently deplete MGM-T activity. ${ }^{18,19}$

\section{Treatment of glioblastoma multiforme in the pre- temozolomide era}

The first treatment step after detection of an intracerebral tumor which provokes suspicion of a central nervous tumor is neurosurgical resection that is as complete as possible.
Many researchers have indicated that the extent of resection is of prognostic value. ${ }^{20}$ Patients with complete macroscopic resection show a prolonged survival. Preoperative infusion of delta-aminolevolinic acid (ALA) marks malignant tissue in fluorescing light which is useful for determining the optimal resection margin intraoperatively. Another technique to improve extent of resection and to avoid neurological deficits is the so called "awake resection". ${ }^{21}$ A combination of ALA-technique and awake-resection is possible and seems to improve surgical approach in selected patients. Although resection techniques have improved it has become evident that even complete resection cannot cure a GBM patient due to disseminated malignant cells surrounding the detectable tumor being able to migrate along the intracerebral tracks and cords. Therefore median survival after resection alone remains at less than 12 months, indicating the clear need for additional treatment options to be developed.

In relation to this, it has been demonstrated that conventional fractionated radiotherapy with an equivalent dose of 54 to $60 \mathrm{gy}$ was able to prolong survival for a median of 3 months, resulting in a median overall survival of 12 months for GBM patients. Nearly all resected and irradiated GBM patients experience a local or distant progression within the central nervous system within 9 months after first surgery, however, metastases outside the central nervous system are rare (less than $1 \%$ ). In the case of relapse, resurgery and/or reirradiation may be offered to the patient followed or replaced by local or systemic chemotherapy based on nitrosoureas such as lomustin, carmustine, or nimustine, or on topoisomerase inhibitors such as teniposide or etoposide..$^{22-27}$ In the US treatment with irinotecan (CPT-11) is an option in this situation. ${ }^{28}$ Interestingly, only drugs administered in vivo are able to penetrate the intact blood-brain barrier and therefore show activity in primary brain tumors.

All of these local and systemic chemotherapeutic options achieved objective response rates of between 0 and $15 \%$, with a median survival benefit of less than 3 months. Overall survival therefore was nearly unchanged at a median of approximately 12 months. Good performance status and younger age were predictive for better response. ${ }^{29}$

The administration of nitrosoureas after completion of irradiation without progression, called "adjuvant" by many authors, demonstrated a modest but consistent improvement of overall survival with the 2-year survival rate increasing from $15 \%$ to $20 \% .^{30,31,27}$ Treatment was given for 6 cycles spaced over 6 months. Continuation of the treatment until progression in the sense of a real maintenance treatment was not examined. 
The low long-term survival prognosis of GBM made further investigation of new drugs necessary. Between 1990 and 2000 the most promising drug under investigation in progressive GBM was temozolomide, an oral alkylating drug with a moderate toxicity profile able to penetrate the intact blood-brain barrier.

\section{Development of temozolomide for glioblastoma multiforme}

Temozolomide was initially developed with the intent to treat patients with metastases to the brain for malignant melanoma but it also showed activity in relapsed GBM patients, encouraging further investigation. ${ }^{32-34}$ Temozolomide is characterized by rapid, nearly complete oral absorption. It converts spontaneously into MTIC (5-(3-methyltriazen-1-yl)imidazol-4-carboxamid), and the active metabolite shows a plasma peak concentration within 30 to 90 minutes after uptake, with a plasma half time of 2 hours. ${ }^{35}$ The cytotoxic activity of temozolomide is explained by O6-methylation of the guanine. O6-methylguanine is mutagenic and able to trigger apoptosis. MGM-T, a DNA-repair enzyme, is involved in removing temozolomideinduced methyl adducts at the same time as it depletes the metabolic capacity of this enzyme. ${ }^{16,36,37}$ Temozolomide was first investigated in an oral 5 day regimen with $250 \mathrm{mg} / \mathrm{m}^{2}$ administered daily, repeated every 4 weeks for 6 cycles. Toxicity was moderate even though a more favorable toxicity profile could be demonstrated for continuous oral treatment at a dosage between 75 and $85 \mathrm{mg} / \mathrm{m}^{2}$ of the body surface. With the exception of an increased risk for pneumocystis carinii pneumonia, antibiotic prophylaxis is recommended in regimens with more than $100 \mathrm{mg}$ daily continuous dosage. There is no documented influence of steroids or enzymeinducing antiepileptic drugs on efficacy of temozolomide.

\section{The situation of relapse}

Yung et al were the first to report the statistically significant superiority of a monotherapy regimen of temozolomide compared to procarbazine at first relapse in patients with GBM and anaplastic astocytoma WHO grade III using a regimen of 200 to $250 \mathrm{mg}$ temozolomide $/ \mathrm{m}^{2}$ body surface orally before breakfast on days 1 to 5 of a 28 days cycle. ${ }^{38,39}$ The objective response rate was about $30 \%$ with a moderate toxicity profile; nausea or vomiting usually was only present on days 1 to 3 of the 5 days of drug incorporation. It was found that 5-HT-3 antagonists were required in some cases, and additionally, grade III and IV hematotoxicity rates were low, with about $15 \%$ of patients affected, and treatment-related myelodysplasia or acute myeloid leucemia being very rare events. ${ }^{40}$ The progression-free survival rate at 6 months was about $40 \%$. The results of Yung et al study were confirmed by other researchers, which led to replacement of nitrosoureas and topoisomerase inhibitor-based regimens for patients with GBM and anaplastic astrocytoma in first relapse. ${ }^{41}$ Temozolomide conventionally dosed with $200 \mathrm{mg} / \mathrm{m}^{2}$ body surface on days 1 to 5 of a 28 day cycle became the most commonly used regimen for GBM and anaplastic astrocytoma patients at first relapse around the world. However, the optimal duration of treatment remains unclear. Some researchers recommend treatment until progression or intolerable toxicity occurs, but the majority of researchers tend to restrict treatment to a maximum of 6 cycles. The standard follow up for these patients includes an MRI scan of the brain every 3 months, however in the last 2 years more and more neurooncologists have been recommending MRI scans every 8 weeks.

In case of relapse after standard dosed temozolomide $(5 / 28)$ treatment, options are limited. While nitrosoureabased regimens have not shown promising activity in this situation, with objective response rates of less than $5 \%$, many researchers have investigated different doseand time-modified temozolomide schedules after standard dosed temozolomide has been administered. ${ }^{42-47}$ Supported by the assumption that continuous treatment with alkylating drugs like temozolomide induces depletion of MGM-T activity, the favorable toxicity profile of temozolomide led to the development of dose-dense, prolonged, and metronomic temozolomide schedules, all of which showing moderate activity when administered after standard temozolomide. ${ }^{48,49}$

The dose-dense or one week on/one week off regimen with temozolomide $150 \mathrm{mg} / \mathrm{m}^{2}$ body surface on days 1 to 7 , repeated on day $15(7 / 14)$ seems to be the most effective regimen, with a $48 \%$ progression-free survival rate at 6 months (PFS6)..$^{50,51}$ The prolonged regimen with 75 to $100 \mathrm{mg} / \mathrm{m}^{2}$ body surface on days 1 to 21, repeated at day 29 (21/28), showed a PFS6 of $30 \%$. The metronomic regimen with daily temozolomide $50 \mathrm{mg} / \mathrm{m}^{2}$ body surface on days 1 to 28 , repeated on day 29 (28/28), demonstrated a PFS6 of $35 \% .^{52}$ Dose- and/or time-intensified temozolomide regimens cause an increased grade III and IV hematotoxicity of $20 \%$ to $40 \%$, with an increased risk of pneumocystis carinii pneumonia and herpes virus infections varying from $0 \%$ to $2 \%$. Antibiotic and antiviral prophylaxis should be considered in these cases. While many neurooncologists seem to prefer dose-dense 
or prolonged temozolomide regimens, there is no proof of the significant superiority of one of these regimens over the standard 5/28 temozolomide so far.

Although standard dosed and dose- and/or time-modified regimens of temozolomide have been found to significantly improve outcome of patients with malignant gliomas, the prognosis of these patients remains unsatisfactory.

\section{Primary treatment situation EORTC/NCIC-protocol}

Wedge and van Rijn collected preclinical data which led to them suggesting a synergistic effect of temozolomide and irradiation therapy. Stupp et al demonstrated the feasibility of concomitant temozolomide and irradiation therapy in newly diagnosed GBM patients. ${ }^{53,54}$ The data encouraged Stupp to initiate a randomized phase III study for newly diagnosed GBM patients in cooperation with the EORTC and the NCIC. ${ }^{5,56}$ Stupp and colleagues compared irradiation therapy with concomitant temozolomide followed by 6 cycles of standard dose temozolomide $(5 / 28)$ versus irradiation therapy alone. This research became a landmark study for the treatment of newly diagnosed GBM patients.

Concomitant temozolomide was dosed at $75 \mathrm{mg} / \mathrm{m}^{2}$ body surface each day, including weekends, during irradiation followed by 6 cycles temozolomide $200 \mathrm{mg} / \mathrm{m}^{2}$ on days 1 to 5, repeated on day 29 (5/28-regimen). Patients received conventionally fractionated irradiation in both arms with a total dose of 55 to $60 \mathrm{gy}$.

The toxicity of temozolomide was moderate with grade III and IV hematotoxicity of $7 \%$ in the concomitant phase and 14\% in the standard dose phase.

Radiotherapy plus temozolomide was statistically significantly superior to radiotherapy alone according to all endpoints, with a consistent difference reported over time. This became the new treatment standard for newly diagnosed GBM patients.

The addition of temozolomide improved patients' median survival (15.6 months versus 12.1 months), 2 year overall survival $(27.2 \%$ versus $10.9 \%), 3$ year overall survival (16.0\% versus 4.4\%), and 5 year overall survival $(9.8 \%$ versus $1.9 \%)$. The superiority of the temozolomide arm continuously improved over time. ${ }^{57}$ In a correlational study on health-related quality of life no negative impact of concomitant temozolomide was found. Additional analysis of the data confirmed good performance status and younger age as predictors for better response to treatment.

Methylation status according to MGM-T gene promoter demonstrated the best results for patients with methylated
MGM-T gene promoter in both treatment arms, as expected, with combined median overall survival in the entire study population of 18.2 years for methylated MGM-T promoter versus 12.2 years for unmethylated MGM-T promoter. According to the treatment arms, the differences became more impressive. Patients with methylated MGM-T promoter had a median survival rate of 21.7 months with combined treatment versus 15.3 months with radiotherapy alone. The 2 year survival rates were $46 \%$ versus $22.7 \%$ respectively, while patients with unmethylated MGM-T promoter had a median survival of 12.7 months with combined treatment versus 11.8 months with radiotherapy alone, and 2 years survival rates were $13.8 \%$ versus $<2 \%$ respectively. ${ }^{58,48}$ As expected, the data indicate that patients with methylated MGM-T gene promoters clearly benefit more from temozolomide but even patients with unmethylated MGM-T gene promoters benefit from temozolomide, especially with long-term survival being possible only in the combined treatment arm.

In the temozolomide era MGM-T gene promoter status again is a clear prognostic factor but still does not have an impact on individualization of treatment. Although there are arguments for the use of a prolonged temozolomide regimen for unmethylated MGM-T gene promoter status, there is no consensus about this overall in the literature.

In a recently published randomized phase II study irradiation plus concomitant temozolomide followed by either dose-dense or metronomic temozolomide was compared in newly diagnosed GBM patients. Toxicity was moderate in both arms but there was no evidence of the superiority of one of the tested arms compared to the historical data of the original EORTC-protocol so far. In this study the prognosis of patients with unmethylated MGM-T gene promoter was better than expected, however the number of patients was too small to reach statistical significance. Further investigation of prolonged temozolomide regimen for patients with unmethylated MGM-T promoter is recommended. ${ }^{59}$

There is evidence in the literature that elderly patients substantially benefit from treatment with temozolomide and despite the side effects, especially an increased rate of neutropenia and thrombocytopenia temozolomide, it should be offered to elderly patients with good performance status as well, especially in the form of a lower dosed continuous regimen. ${ }^{60-63}$

\section{Temozolomide regimen}

1. Standard (5/28):

150 to $200 \mathrm{mg} / \mathrm{m}^{2}$ on days 1 to 5 , repeated on day 29 .

2. Dose-dense (7/7):

$150 \mathrm{mg} / \mathrm{m}^{2}$ on days 1 to 7 , repeated on day 8 . 


\section{Prolonged (21/28):}

75 to $100 \mathrm{mg} / \mathrm{m}^{2}$ on days 1 to 21 , repeated on day 29 .

4. Metronomic (28/28):

$50 \mathrm{mg} / \mathrm{m}^{2}$ continuously on days 1 to 28 , repeated day 29 .

5. Concomitant to irradiation (days 1 to 42/49):

$75 \mathrm{mg} / \mathrm{m}^{2}$ per day for 42 to 49 days during irradiation period.

\section{Further developments and questions}

\section{A. Best temozolomide schedule}

Which temozolomide regimen is the optimal treatment option in which situation remains an unanswered question. Different researchers promote dose-dense, prolonged, or even continuous strategies, especially in case of unmethylated MGM-T promoter status, so an overall consensus regarding which treatment is recommended has not yet been reached. ${ }^{64}$

\section{B. EORTC/NCIC regimen for newly diagnosed astrocytoma WHO grade III}

Many neurooncologists recommend the EORTC/NCIC regimen for newly diagnosed astrocytoma WHO grade III analogue to GBM. Histopathology and clinical characteristics of astrocytoma WHO grade III seem to support this strategy. As phase III studies do not include analyses of treatment of newly diagnosed astrocytoma WHO grade III with temozolomide, these are not included in the current research.

\section{Combination regimen}

The excellent toxicity profile of temozolomide allows for various combinations with antitumor agents. Many studies have been carried out with the purpose of investigating combination therapy of temozolomide with other chemotherapeutic drugs, monoclonal antibodies, and thyrosine kinase inhibitors. None of these combinations, however, have demonstrated statistically significant superiority compared to temozolomide alone. Research must therefore be continued in this area.

\section{Combinations under investigation}

These combinations are under investigation in the first line and relapsed situation (list incomplete):

Temozolomide plus
A. Chemotherapy ${ }^{65-70}$
- $\mathrm{CCNU}$
- Cisplatnum

- Hydroxyurea

- Pegylated liposomal Doxorubicin

- Procarbacin

- Etoposid

- Topotican

- Paclitaxel

- Fotemustine

- Topic BCNU (wafer)

B. Thyrosinekinase-inhibitors (TKI) ${ }^{71-73}$

- Erlotinib

- Gefitinib

- Cediranib

- Cilengitide

- Sunitinib

- Lapatinib

C. Monoclonal antibodies ${ }^{1}$

- Bevacizumab

- Cetuximab

- Panitumumab

D. Further antitumor/metabolic agents ${ }^{74-76}$

- Tamoxifen

- Thalidomide

- Celecoxib

Compared to temozolomide monotherapy, there is currently no evidence that combination with the investigated drugs might be superior. Therefore none of the combination regimens have reached admission status so far.

\section{Summary}

The EORTC/NCIC-regime published by R Stupp in 2005 still is the standard of care for newly diagnosed GBM patients, independent of the methylation status of the MGM-T gene promoter. Age is not a contraindication for treatment with temozolomide, although co-morbidity and performance status have to be considered. Many new treatment options including targeted therapies are evolving and will sooner or later optimize the treatment for patients with malignant brain tumors. For temozolomide-naive GBM and astrocytoma grade III patients with disease progression, temozolomide is still the treatment of choice outside of clinical studies. An overall consensus on a recommendation for the schedule of choice has not yet been achieved, although so far the 5 out of 28 days regimen $(5 / 28)$ is the standard of care in most countries.

Patients with disease progression after standard temozolomide $(5 / 28)$ are candidates for clinical studies. Outside of clinical studies dose-dense (7/7), prolonged $(21 / 28)$, or metronomic $(28 / 28)$ temozolomide, or alternatively 
nitrosourea-based regimens can be an option. Individualized treatment options including resurgery, reirradiation, or a combination of drugs should be discussed with the patient based on individual characteristics and relevant literature.

As long as potentially effective treatment alternatives are available, 6-weekly (or 8 at the most) MRI scans are recommended to detect tumor progression in time to be able to change treatment before deterioration of performance status makes further treatment impossible.

Well designed fast track studies (endpoint PFS6 or shorter, ie, PFS3) are needed to identify treatment strategies with real treatment potential within the growing offer of old, new, and up-coming drugs and their combinations. These studies should be followed by slow track studies (endpoint PFS12 or survival) to define the role of these strategies within the established treatment options.

Although patent protection of temozolomide ran out in 2009 investigation of temozolomide as a substantial part of the treatment of malignant brain tumors should be continued. In particular, the role of lower dosed continuous regimen with or without drug combinations and the role of temozolomide for newly diagnosed astrocytoma grade III and low grade glioma needs to be determined.

\section{Disclosure}

The author reports no conflicts of interest in this work.

\section{References}

1. Louis DN, Ohgaki H, Wiestler OD, Cavenee WK, editors. WHO classification of tumours of the central nervous system. Lyon, France: IARC; 2007.

2. Eoli M, Menghi F, Bruzzone MG, et al. Methylation of O6-methylguanine DNA methyltransferase and loss of heterozigosity on $19 q$ and/or $17 p$ are overlapping features of secondary glioblastomas with prolonged survival. Clin Cancer Res. 2007;13:2606-2613.

3. Abrey LE, Louis DN, Paleologos N, et al. Survey of treatment recommendations for anaplastic oligodendroglioma. Neurooncol. 2007;9:314-318.

4. Cairncross G, Seiferheld W, Shaw E, et al. Phase III trial of chemotherapy plus radiotherapy compared with radiotherapy alone for pure and mixed anaplastic oligodendroglioma: Intergroup Radiation Therapy Oncology Group Trial 9402. J Clin Oncol. 2006;24: 2707-2714.

5. Hanisch UK, Kettenmann H. Microglia: active sensor and versatile effector cells in normal and pathologic brain. Nat Neurosci. 2007;10: 1387-1397.

6. Carapancea M, Alexandru O, Fetea A, et al. Growth factor receptors signaling in glioblastoma cells: therapeutic implications. J Neurooncol. 2009;92:137-147.

7. Guha A, Dashner K, Black PM, Wagner JA, Stiles CD. Expression of PDGF and PDGF receptors in human astrocytoma operation specimens supports the existence of an autocrine loop. Int J Cancer. 1995;60: 168-173.

8. Hermanson M, Funa K, Hartman M, et al. Plateletderived growth factor and its receptors in human glioma tissue: expression of messenger RNA and protein suggests the presence of autocrine and paracrine loops. Cancer Res. 1992;52:3213-3219.
9. Jain RK, di Tomaso E, Duda DG, et al. Angiogenesis in brain tumor. Nat Rev Neurosci. 2007;8:610-622.

10. Pelloski C, Ballman K, Furth A, et al. Epidermal growth factor receptor variant III status defines clinically distinct subtypes of glioblastoma. $J$ Clin Oncol. 2007;25:2288-2294.

11. Plate KH, Breier G, Weich HA, Risau W. Vascular endothelial growth factor is a potential tumor angiogenesis factor in human gliomas in vivo. Nature. 1992;359:845-848.

12. Wong AJ, Bigner SH, Bigner DD, et al. Increased expression of the epidermal growth factor receptor gene in malignant gliomas is invariably associated with gene amplification. Proc Natl Acad Sci U S A. 1987;84:6899-6903.

13. Gladson CL, Cheresh DA. Glioblastoma expression of vitronectin and the avh3 integrin. Adhesion mechanism for transformed glial cells. J Clin Invest. 1991;88:1924-1932.

14. Maher EA, Brennan C, Wen PY, et al. Marked genomic differences characterize primary and secondary glioblastoma subtypes and identify two distinct molecular and clinical secondary glioblastoma entities. Cancer Res. 2006;66:11502-11513.

15. Parsons DW, Jones S, Zhang X, et al. An integrated genomic analysis of human glioblastoma multiforme. Science. 2008;321: 1807-1812.

16. Friedman H, McLendon R, Kerby T, et al. DNA mismatch repair and O6-alkylguanine-DNA alkyltransferase analysis and response to temodal in newly diagnosed malignant glioma. J Clin Oncol. 1998; 16:3851-3857.

17. Sarkaria JN, Kitange GJ, James CD, et al. Mechanisms of chemoresistance to alkylating agents in malignant glioma. Clin Cancer Res. 2008;14:2900-2908.

18. Beith J, Cook R, Robinson BLJ, et al. Modulation of resistance to BCNU by depleting MGMT activity with procarbazine in patients with relapsed high grade gliomas. Proc Am Assoc Cancer Res. 1997;16:386.

19. Friedman HS. Can O6-alkylguanine-DNA alkyltransferase depletion enhance alkylator activity in the clinic? Clin Cancer Res. 2000;6:2967-2968.

20. Sanai N, Berger MS. Glioma extent of resection and its impact on patient outcome. Neurosurgery. 2008;62:753-766.

21. Stummer W, Pichlmeier U, Meinel T, et al. Fluorescence-guided surgery with 5-aminolevulinic acid for resection of malignant glioma: a randomized controlled multicentre phase III trial. Lancet Oncol. 2006;7:392-401.

22. Brem H, Piantadosi S, Burger PC, et al. Placebo-controlled trial of safety and efficacy of intraoperative controlled delivery by biodegradable polymers of chemotherapy for recurrent gliomas. The Polymer-brain Tumor Treatment Group. Lancet. 1995;345:1008-1012.

23. Guyotat J, Signorelli F, Frappaz D, et al. Is reoperation for recurrence of glioblastoma justified? Oncol Rep. 2000;7:899-904.

24. Hau P, Baumgart U, Pfeifer K, Bock A, et al. Salvage therapy in patients with glioblastoma: is there any benefit. Cancer. 2003;98:2678-2686.

25. Patel M, Siddiqui F, Jin JY, et al. Salvage reirradiation for recurrent glioblastoma with radiosurgery: radiographic response and improved survival. J Neurooncol. 2009;92:185-191.

26. Westphal M, Hilt DC, Bortey E, et al. A phase III trial of local chemotherapy with biodegradable carmustine (BCNU) wafers (Gliadel wafers) in patients with primary malignant glioma. Neurooncol. 2003;5:79-88.

27. Wolff JE, Berrak S, Koontz Webb SE, et al. Nitrosourea efficacy in high-grade glioma: a survival gain analysis summarizing 504 cohorts with 24193 patients. $J$ Neurooncol. 2008;88:57-63.

28. Chen TC, Su S, Fry D, Liebes L. Combination therapy with irinotecan and protein kinase $\mathrm{C}$ inhibitors in malignant glioma. Cancer. 2003;97:2363-2373.

29. Brandes AA, Ermani M, Turazzi S, et al. Procarbazine and high-dose tamoxifen as a second- line regimen in recurrent high-grade gliomas: a phase ii study. J Clin Oncol. 1999;17:645-650. 
30. van den Bent MJ, Carpentier AF, Brandes AA, et al. Adjuvant procarbazine, lomustine and vincristine improves progression-free survival but not overall survival in newly diagnosed anaplastic oligodendrogliomas: a randomized European Organization for Research and Treatment of Cancer phase III trial. J Clin Oncol. 2006;24:2715-2722.

31. Walker MD, Green SB, Byar DP, et al. Randomized comparison of radiotherapy and nitrosoureas for the treatment of malignant glioma after surgery. N Engl J Med. 1980;303:1323-1329.

32. Biasco G, Pantaleo MA, Casadei S. Treatment of brain metastases of malignant melanoma with temozolomide. $N$ Engl J Med. 2001;345:621-622.

33. Brada M, Judson I, Beale P, et al. Phase I dose-excalation and pharmacokinetic study of temozolomide (SCH 52365) for refractory or relapsing malignancies. Br J Cancer. 1999;81:1022-1030.

34. O'Reilly SM, Newlands ES, Glaser MG, et al. Temozolomide: a new oral cytotoxic chemotherapeutic agent with promising activity against primary brain tumors. Eur J Cancer. 1993;29:940-942.

35. Newlands ES, Stevens MF, Wedge SR, et al. Temozolomide. A review of its discovery, chemical properties, pre-clinical development and clinical trials. Cancer Treat Rev. 1997;23:35-61.

36. Gerson SL. Clinical relevance of MGMT in the treatment of cancer. J Clin Oncol. 2002;20:2388-2399.

37. Paz MF, Yaya-Tur R, Rojas-Marcos I, et al. CpG island hypermethylation of the DNA repair enzyme methyltransferase predicts response to temozolomide in primary gliomas. Clin Cancer Res. 2004; 10:4933-4938

38. Yung WK, Albright RE, Olson J, et al. A phase II study of temozolomide vs procarbazine in patients with glioblastoma multiforme at first relapse. Br J Cancer. 2000;83:588-593.

39. Yung WK, Prados MD, Yaya-Tur R, et al. Multicenter phase II trial of temozolomide in patients with anaplastic astrocytoma or anaplastic oligoastrocytoma at first relapse, Temodal Brain Tumor Group. J Clin Oncol. 1999; 17:2762-2771.

40. Kim SH, Park TS, Lee ST, et al. Therapy-related myelodysplasia/acute myeloid leucemia after treatment with temozolomide in a patient with glioblastoma multiforme. Ann Clin Lab Sci. 2009;39:392-398.

41. Brada M, Hoang-Xuan K, Rampling R, et al. Multicenter phase II trial of temozolomide in patients with glioblastoma multiforme at first relapse. Ann Oncol. 2001;12:259-266.

42. Franceschi E, Omuro AM, Lassman AB, et al. Salvage temozolomide for prior temozolomide responders. Cancer. 2005;104:2473-2476.

43. Happold C, Roth P, Wick W, et al. ACNU-based chemotherapy for recurrent glioma in the temozolomide era. J Neurooncol. 2009;92:45-48.

44. Jauch T, Hau P, Bogdahn U. Re-challenge with temozolomide (TMZ) at recurrence in high-grade gliomas. Proc ASCO. 2007;25:83s.

45. Kappelle AC, Postma TJ, Taphoorn MJB, et al. PCV chemotherapy for recurrent glioblastoma multiforme. Neurology. 2001;56:118-120.

46. Kong DS, Lee JI, Kim WS, et al. A pilot study of metronomic temozolomide treatment in patients with recurrent temozolomide-refractory glioblastoma. Oncol Rep. 2006;16:1117-1121.

47. Triebels VH, Taphoorn MJ, Brandes AA, et al. Salvage PCV chemotherapy for temozolomide-resistant oligodendrogliomas. Neurology. 2004;63:904-906.

48. Hegi ME, Liu L, Herman J, et al. Correlation of O6-Methylguanine Methyltransferase MGMT promoter methylation with clinical outcomes in glioblastoma and clinical strategies to modulate MGMT activity. J Clin Oncol. 2008;26:4189-4199.

49. Tolcher AW, Gerson SL, Denis L, et al. Marked inactivation of O6alkylguanine-DNA-alkyltansferase activity with protracted temozolomide schedules. Br J Cancer. 2003;88:1004-1011.

50. Wick W, Steinbach JP, Kuker WM, et al. One week on/one week off: a novel active regimen for temozolomide for recurrent glioblastoma. Neurol. 2004;62:2113-2115.

51. Wick A, Felsberg J, Steinbach JP, et al. Efficacy and tolerability of temozolomide in an alternating weekly regimen in patients with recurrent glioma. J Clin Oncol. 2007;25:3357-3361.
52. Brandes AA, Tosoni A, Cavallo G, et al. Temozolomide 3 weeks on and 1 week off as first-line therapy for recurrent glioblastoma: phase II study from gruppo italiano cooperative di neuro-oncologia (GICNO). Br J Cancer. 2006;95:1155-1160.

53. van Rijn J, Heimans JJ, van den Berg J, et al. Survival of human glioma cells treated with various combination of temozolomide and x-rays. Int J Radiat Oncol Biol Phys. 2000;47:779-784.

54. Wedge SR, Porteous JK, Glaser MG, et al. In vitro evaluation of temozolomide combined with X-irradiation. Anticancer Drugs. 1997;8:92-97.

55. Stupp R, Hegi ME, van den Bent MJ, et al; European Organisation for Research and Treatment of Cancer Brain Tumor and Radiotherapy Groups, National Cancer Institute of Canada Clinical Trials Group. Changing paradigms - an update on the multidisciplinary management of malignant glioma. Oncologist. 2006;11:165-180.

56. Stupp R, Mason WP, van den Bent MJ, et al. Radiotherapy plus concomitant and adjuvant temozolomide for glioblastoma. $N$ Engl J Med. 2005;352:987-996.

57. Stupp R, Hegi M, Mason W, et al. Effects of radiotherapy with concomitant and adjuvant temozolomide versus radiotherapy alone on survival in glioblastoma in a randomised phase III study: 5-year analysis of the EORTC-NCIC trial. Lancet Oncol. 2009;10:459-466.

58. Hegi M, Diserens A-C, Gorlia T, et al. MGMT gene silencing and benefit from temozolomide in glioblastoma. $N$ Engl J Med. 2005;352:997-1003.

59. Clarke JL, Iwamoto FM, Sul J, et al. Randomized phase II trial of chemoradiotherapy followed by either dose-dense or metronomic temozolomide for newly diagnosed Glioblastoma. J Clin Oncol. 2009;27:3861-3867.

60. Brandes AA, Franceschi E, Tosoni A, et al. Temozolomide concomitant and adjuvant to radiotherapy in elderly patients with glioblastoma: correlation with MGMT promoter methylation status. Cancer. 2009; 115:3512-3518.

61. Chamberlain MC, Chalmers LM. A pilot study of primary temozolomide chemotherapy and deferred radiotherapy in elderly patients with glioblastoma. J Neurooncol. 2007;82:207-209.

62. Iwamoto FM, Coeper AR, Reiner AS, et al. Glioblastoma in the elderly: The Memorial Sloan-Kettering Cancer Center experience (1997-2007). Cancer. 2009;115:3758-3766.

63. Minniti G, De Sanelis V, Muni R, et al. Hypofractionated radiotherapy followed by adjuvant chemotherapy with temozolomide in elderly patients with glioblastoma. J Neurooncol. 2009;91:95-100.

64. Hau P, Koch D, Hundsberger T, et al. Safety and feasibility of long-term temozolomide treatment in patients with high grade glioma. Neurology. 2007;68:688-690.

65. Glas M, Happold C, Rieger J, et al. Long-term survival of patients with glioblastoma treated with radiotherapy and lomustine plus temozolomide. J Clin Oncol. 2009;27:1257-1261.

66. Korones DN, Benita-Weiss M, Coyle TE, et al. Phase I study of temozolomide and escalating doses of oral etoposide for adults with recurrent malignant glioma. Cancer. 2003;97:1963-1968.

67. McGirt MJ, Than KD, Weingart JD, et al. Gliadel (BCNU) wafer plus concomitant temozolomide therapy after primary resection of glioblastoma multiforme. J Neurosurg. 2009;110:583-588.

68. Prados MD, Yung WKA, Fine HA, et al. Phase 2 study of BCNU and temozolomide for recurrent glioblastoma multiforme: North American Brain Tumor Consortium study. Neurooncol. 2004;6:33-37.

69. Raizer JJ, Malkin MG, Kleber M, et al. Phase 1 study of 28-day, lowdose temozolomide and BCNU in the treatment of malignant gliomas after radiation therapy. Neurooncol. 2004;6:247-252.

70. Silvani A, Eoli M, Salmaggi A, et al. Phase II trial of cisplatin plus temozolomide, in recurrent and progressive malignant glioma patients. J Neurooncol. 2004;66:203-208.

71. Nicholas MK, Lukas RV, Jafri NF, et al. Epidermal growth factor receptor-mediated signal transduction in the development and therapy of gliomas. Clin Cancer Res. 2006;12:7261-7270. 
72. Prados M, Chang S, Butowski C, et al. Phase II study of erlotinib plus temozolomide during and after radiation therapy in patients with newly diagnosed glioblastoma multiforme or gliosarcoma. J Clin Oncol. 2009;27:579-584.

73. Prados MD, Lamborn KR, Chang S, et al. Phase I study of erlotinib $\mathrm{HCI}$ alone and combined with temozolomide in patients with stable or recurrent malignant glioma. Neurooncol. 2006;8:67-78.

74. Lai A, Filka E, McGibbon B, et al. Phase II pilot study of bevacizumab in combination with temozolomide and regional radiation therapy for up-front treatment of patients with newly diagnosed glioblastoma multiforme: interim analysis of safety and tolerability. Int J Radiat Oncol Biol Phys. 2008;71:1372-1380.
75. Gupta V, Su YS, Wang W, et al. Enhancement of glioblastoma cell killing by combination treatment with temozolomide and tamoxifen or hypericin. Neurosurg Focus. 2006;20(4):E20.

76. Spence AM, Peterson RA, Scharnhorst JD, et al. Phase II study of concurrent continous temozolomide (TMZ) and tamoxifen (TMX) for recurrent malignant astrocytic gliomas. J Neurooncol. 2004;70:91-95.

\section{Publish your work in this journal}

OncoTargets and Therapy is an international, peer-reviewed, open access journal focusing on the pathological basis of all cancers, potential targets for therapy and treatment protocols employed to improve the management of cancer patients. The journal also focuses on the impact of management programs and new therapeutic agents and protocols on

\section{Dovepress}

patient perspectives such as quality of life, adherence and satisfaction. The manuscript management system is completely online and includes a very quick and fair peer-review system, which is all easy to use. Visit http://www.dovepress.com/testimonials.php to read real quotes from published authors.

Submit your manuscript here: http://www.dovepress.com/oncotargets-and-therapy-journal 